\title{
Reliability of the velocity achieved during the last repetition of sets to failure and its association with the velocity of the 1- repetition maximum
}

\author{
Amador García-Ramos ${ }^{\text {Corresp., 1, 2, Danica Janicijevic }}{ }^{3}$, Jorge M González-Hernández ${ }^{4}$, Justin W. L. Keogh ${ }^{5,6,7,8}$, \\ Jonathon Weakley ${ }^{9,10}$ \\ ${ }^{1}$ Department of Sports Sciences and Physical Conditioning, Universidad Católica de la Santísima Concepción, Concepción, Chile \\ 2 Department of Physical Education and Sport, Faculty of Sport Sciences, University of Granada, Granada, Spain \\ 3 Faculty of sport and physical education, The Research centre, University of Belgrade, Belgrade, Serbia \\ 4 Faculty of Health Science, Universidad Europea de Canarias, La Orotava, Tenerife, Spain \\ 5 Faculty of Health Sciences and Medicine, Bond University, Gold Coast, Australia \\ 6 Sports Performance Research Centre New Zealand, AUT University, Auckland, New Zealand \\ 7 Cluster for Health Improvement, Faculty of Science, Health, Education and Engineering, University of the Sunshine Coast, Gold Coast, Australia \\ 8 Kasturba Medical College, Manipal Academy of Higher Education, Manipal, Karnataka, India \\ 9 School of Behavioural and Health Sciences, Australian Campus University, Brisbane, Australia \\ 10 Carnegie Applied Rugby Research (CARR) centre, Institute for Sport, Physical Activity and Leisure, Leeds Beckett University, Leeds, United Kingdom \\ Corresponding Author: Amador García-Ramos \\ Email address: amagr@ugr.es
}

Background. This study aimed to determine the reliability of the velocity achieved during the last repetition of sets to failure $\left(V_{\text {last }}\right)$ and the association of $V_{\text {last }}$ with the velocity of the 1-repetition maximum $\left(\mathrm{V}_{1 \mathrm{RM}}\right)$ during the paused and touch-and-go bench press (BP)

exercises performed in a Smith machine. Methods. Ninety-six healthy men participated in this study that consisted of two testing sessions. A single BP variant (paused BP or touchand-go BP) was evaluated on each session in a randomized order. Each session consisted of an incremental loading test until reaching the $1 \mathrm{RM}$, followed by two sets of repetitions to failure against a load ranging from $75-90 \%$ of $1 R M$. Results. The reliability of $V_{\text {last }}$ was unacceptable for both BP variants ( $C V>18.3 \%$, ICC $<0.60$ ). The correlations between $\mathrm{V}_{1 \mathrm{RM}}$ and $\mathrm{V}_{\text {last }}$ were small for the paused BP $(r=0.18)$ and moderate for the touch-and-go BP ( $r$ $=0.37)$. Conclusions. Although these results suggest that $V_{\text {last }}$ could be a better indicator of the minimal velocity threshold than $\mathrm{V}_{1 \mathrm{RM}}$, the low reliability of $\mathrm{V}_{\text {last }}$ and the similar values of $V_{\text {last }}$ for both BP variants suggest that a standard $V_{1 R M}$ should be used to estimate the 1RM from the individualized load-velocity relationship. 
1 Reliability of the velocity achieved during the last

2 repetition of sets to failure and its association with the

3 velocity of the 1-repetition maximum

Amador Garcia-Ramos ${ }^{1,2}$, Danica Janicijevic ${ }^{3}$, Jorge M González-Hernández ${ }^{4}$, Justin W. L. Keogh ${ }^{5,6,7,8}$, Jonathon Weakley ${ }^{9,10}$

${ }^{1}$ Department of Sports Sciences and Physical Conditioning, Universidad Católica de la Santísima Concepción, Concepción, Chile

${ }^{2}$ Department of Physical Education and Sport, Faculty of Sport Sciences, University of Granada, Granada, Spain

3 University of Belgrade, Faculty of Sport and Physical Education, The Research Centre, Belgrade, Serbia.

${ }^{4}$ Faculty of Health Science, Universidad Europea de Canarias, La Orotava, Tenerife, Spain

${ }^{5}$ Faculty of Health Sciences and Medicine, Bond University, Gold Coast, Australia ${ }^{6}$ Sports Performance Research Centre New Zealand, AUT University, Auckland, New Zealand

${ }^{7}$ Cluster for Health Improvement, Faculty of Science, Health, Education and Engineering, University of the Sunshine Coast, Gold Coast, Australia

${ }^{8}$ Kasturba Medical College, Manipal Academy of Higher Education, Manipal, Karnataka, India

9 School of Behavioural and Health Sciences, Australian Campus University, Brisbane, Australia

${ }^{10}$ Carnegie Applied Rugby Research (CARR) centre, Institute for Sport, Physical

Activity and Leisure, Leeds Beckett University, Leeds, UK

\section{Corresponding author:}

Amador García-Ramos

Av. Alonso de Ribera 2850, Concepción, Bío Bío, Chile

E-mail address: amgarcia@ucsc.cl 


\section{Abstract}

Background. This study aimed to determine the reliability of the velocity achieved during the last repetition of sets to failure $\left(\mathrm{V}_{\text {last }}\right)$ and the association of $\mathrm{V}_{\text {last }}$ with the velocity of the 1-repetition maximum $\left(\mathrm{V}_{1 \mathrm{RM}}\right)$ during the paused and touch-and-go bench press (BP) exercises performed in a Smith machine.

Methods. Ninety-six healthy men participated in this study that consisted of two testing sessions. A single BP variant (paused BP or touch-and-go BP) was evaluated on each session in a randomized order. Each session consisted of an incremental loading test until reaching the $1 \mathrm{RM}$, followed by two sets of repetitions to failure against a load ranging from $75-90 \%$ of $1 \mathrm{RM}$.

Results. The reliability of $V_{\text {last }}$ was unacceptable for both BP variants (CV $>18.3 \%$, ICC $<0.60)$. The correlations between $\mathrm{V}_{1 \mathrm{RM}}$ and $\mathrm{V}_{\text {last }}$ were small for the paused $\mathrm{BP}(r=0.18)$ and moderate for the touch-and-go BP $(r=0.37)$.

Conclusions. Although these results suggest that $\mathrm{V}_{\text {last }}$ could be a better indicator of the minimal velocity threshold than $V_{1 R M}$, the low reliability of $V_{\text {last }}$ and the similar values of $V_{\text {last }}$ for both BP variants suggest that a standard $V_{1 R M}$ should be used to estimate the $1 \mathrm{RM}$ from the individualized load-velocity relationship.

Keywords: bench press, linear position transducer, minimal velocity threshold, strength testing, velocity-based training. 
59

60

61

62

63

64

65

66

67

68

69

70

71

72

73

74

75

76

77

78

79

80

81

82

83

84

85

86

87

88

89

90

91

92

93

94

95

96

97

98

99

100

101

102

103

104

\section{Introduction}

Movement velocity has been proposed as an accurate variable for estimating the 1repetition maximum (1RM) during a number of resistance training exercises (GarcíaRamos \& Jaric, 2018; McBurnie et al., 2019). Early studies proposed generalized loadvelocity $(\mathrm{L}-\mathrm{V})$ relationship equations to estimate the percentage of $1 \mathrm{RM}$ from the velocity value recorded against a submaximal load lifted with maximal effort (GonzálezBadillo \& Sánchez-Medina, 2010). The basic premise of generalized L-V relationship equations is that a given velocity represents the same percentage of 1RM for all individuals. However, this premise has been refuted in subsequent studies which have shown a greater accuracy in the estimation of the percentage of 1RM using individualized L-V relationships (García-Ramos \& Jaric, 2018; García-Ramos et al., 2018; McBurnie et al., 2019). This is also demonstrated by the between-subject variability in the velocity associated with a given percentage of $1 \mathrm{RM}$ being higher than the within-subject variability (Pestaña-Melero et al., 2018). On the basis of these results, an increasing number of studies have recently been conducted to refine the testing procedure of the individualized $\mathrm{L}-\mathrm{V}$ relationship.

Assessment of individualized $\mathrm{L}-\mathrm{V}$ relationships requires the recording of movement velocity against at least 2 submaximal loads and, subsequently, the 1RM can be estimated through a linear regression as the load associated with the velocity of the 1RM ( $V_{1 R M}$ or minimal velocity threshold) (García-Ramos \& Jaric, 2018; GarcíaRamos et al., 2018; McBurnie et al., 2019). However, one of the challenges associated with the use of individualized L-V relationships for predicting the $1 \mathrm{RM}$ is how to select the minimal velocity threshold. Previous studies have selected the minimal velocity threshold as either the individualized $\mathrm{V}_{1 \mathrm{RM}}$ (Banyard, Nosaka \& Haff, 2017; Ruf, Chery \& Taylor, 2018) or a general $V_{1 R M}$ for all subjects (Garcia-Ramos et al., 2019; GarcíaRamos et al., 2018). The assessment of the individualized $\mathrm{V}_{1 \mathrm{RM}}$ is associated with at least 2 problems: (I) the individual is required to perform a lift against the 1RM load, and (II) the individual $\mathrm{V}_{1 \mathrm{RM}}$ has been demonstrated to be an unreliable metric for a number of exercises such as the back squat (coefficient of variation [CV] $=22.5 \%$, intraclass correlation coefficient [ICC] $=0.42$ ) (Banyard, Nosaka \& Haff, 2017), deadlift (CV = $15.7 \%, I C C=0.63$ ) (Ruf, Chery \& Taylor, 2018), Smith machine bench press (BP) (CV $=13.9-15.7 \%$, ICC = 0.54-0.64) (Pestaña-Melero et al., 2018), or bench pull (CV = $6.36 \%$, ICC $=0.18$ ) (García-Ramos et al., 2019). Therefore, it would be of interest to investigate whether the minimal velocity threshold (i.e. velocity value used to estimate the 1RM from the individualized L-V relationship) can be obtained with a higher reliability using other approaches that do not require the individual to perform a lift against the 1RM load.

An alternative approach for determining the minimal velocity threshold could be the assessment of the velocity of the last repetition performed during a set to failure $\left(V_{\text {last }}\right)$ (Lake et al., 2017). This approach is supported by the results of Izquierdo et al. (2006) who did not find significant differences between the individual $V_{1 R M}$ and the $V_{\text {last }}$ collected against 4 submaximal loads $(60,65,70$ and $75 \%$ of $1 \mathrm{RM})$ during the BP and parallel back squat exercises performed in a Smith machine. A recent review has suggested that the accuracy of the individualized L-V relationship for predicting the 1RM 
105 is higher for upper-body (e.g. BP or bench pull) compared to lower-body exercises (e.g. 106 squat or deadlift), while the BP is the exercise most explored in velocity-based training

107

108

109

110

111

112

113

114

115

116

117

118

119

120

121

122

123

124

125

126

127

128

129

130

131

132

133

134

135

136

137

138

139

140

141

142

143

144

145

146

147

148

149

150 research (McBurnie et al., 2019). The 2 main variants of the BP exercise examined in the scientific literature are the paused BP (a pause is introduced between the eccentric and concentric phases) and the touch-and-go BP (the concentric phase is performed immediately after the eccentric phase) (García-Ramos, Pestaña-Melero, Pérez-Castilla, Rojas, \& Haff, 2018; Pallarés, Sánchez-Medina, Pérez, De La Cruz-Sánchez, \& MoraRodriguez, 2014; Pérez-Castilla, Comfort, McMahon, Pestaña-Melero, \& GarcíaRamos, 2018). García-Ramos et al. (2018) revealed a comparable $V_{1 \mathrm{RM}}$ for the paused $\operatorname{BP}(0.168 \pm 0.026 \mathrm{~m} / \mathrm{s})$ and touch-and-go BP $(0.178 \pm 0.030 \mathrm{~m} / \mathrm{s})(P=0.232)$ performed in a Smith machine, while the $\mathrm{V}_{1 \mathrm{RM}}$ was poorly related between the $\mathrm{BP}$ variants $(r=-0.11, P=0.554)$. However, no study has examined the reliability of $V_{\text {last }}$ or the association between $V_{\text {last }}$ and $V_{1 R M}$. Therefore, a comprehensive examination of the behavior of $V_{\text {last }}$ during the BP exercise is needed to clarify whether $V_{\text {last }}$ may provide useful information for increasing the accuracy in the estimation of the 1RM through the individualized L-V relationship.

To address the aforementioned gaps in the literature, the main aim of the present study was to assess the reliability of $V_{\text {last }}$ and the association between $V_{\text {last }}$ and $V_{1 R M}$ during the paused and touch-and-go BP exercises performed in a Smith machine. In addition, we aimed to determine the effect of the number of repetitions performed to failure on $V_{\text {last }}$ and the effect of $1 R M$ strength on $V_{1 R M}$ and $V_{\text {last. }}$. Our main hypothesis was that, regardless of the BP variant, $V_{\text {last }}$ would present a low level of reliability and it would be poorly correlated with $\mathrm{V}_{1 \mathrm{RM}}$. We also hypothesized that no significant correlations would be observed between $V_{\text {last }}$ and the number of repetitions performed to failure, while the $1 R M$ strength would be negatively associated with $V_{1 R M}$ and $V_{\text {last. }}$.

\section{Materials \& Methods Participants}

Ninety-six healthy men volunteered to participate in this study (age $=20.8 \pm 3.4$ years [range $=18-38$ years]; body height $=1.73 \pm 0.06 \mathrm{~m}$; body mass $=75.3 \pm 15.7 \mathrm{~kg}$; paused BP 1RM $=62.3 \pm 17.6 \mathrm{~kg}$; touch-and-go BP $1 \mathrm{RM}=66.5 \pm 18.4 \mathrm{~kg}$; resistance training experience $=1.3 \pm 2.4$ years [range $=0-10$ years]). Eighty-six participants completed both testing sessions, 7 participants only performed the paused $\mathrm{BP}$, and 3 participants only performed the touch-and-go BP. 10 participants only performed one $\mathrm{BP}$ variant because they reported not to be interested in attending a second testing session. All participants without resistance training experience were first year sport science students and they were familiarized with the BP exercise during several sessions before the onset of the study. Prior to study initiation, participants were informed of the study procedures and provided written informed consent. Additionally, participants were instructed to avoid any strenuous exercise for the 48 hours before each testing session. The study protocol adhered to the tenets of the Declaration of Helsinki and was approved by the Institutional Review Board of the University of Granada (491/CEIH/2018).

\section{Study design}

Peer] reviewing PDF | (2019:11:43603:1:1:NEW 22 Jan 2020) 
151 A randomized crossover design was used to comprehensively examine the relationship

152 between $V_{1 R M}$ and $V_{\text {last }}$ during the BP exercise performed in a Smith machine.

153 Participants came to the laboratory on 2 occasions separated by $72-96$ hours. A single

154 BP variant (paused BP or touch-and-go BP) was evaluated on each session in a

155

156

157 randomized order. Each session consisted of an incremental loading test until reaching the $1 \mathrm{RM}$, followed by 2 sets of repetitions to failure against a load ranging from the $75 \%$ of $1 \mathrm{RM}$ to the $90 \%$ of $1 \mathrm{RM}$. The same participant performed the 2 sets against the same load for reliability purposes, while the prescribed relative load (\% of $1 \mathrm{RM})$ differed between participants to explore the effect of the number of repetitions performed on $V_{\text {last }}$ Participants were instructed to perform all repetitions at the maximum possible velocity and the mean concentric velocity ( $\mathrm{MCV}$, average velocity value from the start of the concentric phase until the velocity of the barbell was $0 \mathrm{~m} \cdot \mathrm{s}^{-1}$ ) of the barbell was recorded with a linear velocity transducer (T-Force System; Ergotech, Murcia, Spain). The 2 sessions for the same participant were held at the same time of the day $( \pm 1$ hour) to minimize the influence of the circadian rhythm on physical performance.

\section{Procedures}

Both testing sessions began with a standardized warm-up which consisted of 5 minutes of jogging, dynamic stretching, arm and shoulder mobilization, and 1 set of 10 repetitions with an external load of $20 \mathrm{~kg}$ (mass of the unloaded Smith machine barbell) in the tested BP variant. The initial load of the incremental loading test was $20 \mathrm{~kg}$ and it was increased in $10 \mathrm{~kg}$ until the MCV of the barbell was lower than $0.50 \mathrm{~m} \cdot \mathrm{s}^{-1}$. From that moment, the load was increased in increments of $1-5 \mathrm{~kg}$ until the $1 \mathrm{RM}$ load was achieved. During the incremental loading test, 2 repetitions were performed when the MCV was higher than $0.50 \mathrm{~m} \cdot \mathrm{s}^{-1}$, and only 1 repetition when the MCV was lower than $0.50 \mathrm{~m} \cdot \mathrm{s}^{-1}$. The rest between sets was 3 minutes when the MCV was higher than 0.50 $\mathrm{m} \cdot \mathrm{s}^{-1}$ and 5 minutes when the MCV was lower than $0.50 \mathrm{~m} \cdot \mathrm{s}^{-1}$.

Ten minutes after the 1RM assessment, participants performed 2 sets of repetitions to failure with a load ranging between $75 \%$ and $90 \%$ of the previously determined $1 \mathrm{RM}$. The 2 sets were separated by 10 minutes. Participants performed the 2 sets with the same load for reliability purposes, but the relative load differed between participants to explore the effect of the number of repetitions performed on $V_{\text {last. }}$. Participants were instructed to perform all repetitions at the maximum possible velocity. Two trained spotters were present during the test for safety reasons and to encourage participants to lift the maximum possible load during the 1RM assessment and to perform the maximum possible number of repetitions during the sets of repetitions to failure. The BP was performed in a Smith machine (Ffittech, Taiwan, China), while a linear velocity transducer, which sampled the velocity of the barbell at $1,000 \mathrm{~Hz}$, was used to collect the MCV of all repetitions. Note that the MCV has been reported to be the most accurate velocity variable for determining the load-velocity relationship during the BP exercise (García-Ramos et al., 2018c).The 2 BP variants evaluated in the present study are described below:

- Paused BP: Participants initiated the task holding the barbell with their arms fully extended. They lowered the barbell at a self-selected velocity until the barbell made 
197 contact with their chest, waited with the barbell on the chest for 2 seconds, and on the

198

199

200

201

202

203

204

205

206

207

208

209

210

211

212

213

214

215

216

217

218

219

220

221

222

223

224

225

226

227

228

229

230

231

232

233

234

235

236

237

238

239

240

241

242

word 'Go!' performed a purely concentric action at maximum possible velocity until their arms were fully extended.

- Touch-and-go BP: Participants initiated the task holding the barbell with their arms fully extended. They were instructed to lower the barbell until it touched the chest and then immediately perform the concentric phase at the maximum possible velocity.

\section{Statistical analysis}

Descriptive data are presented as means and standard deviations. The normal distribution of the data was confirmed by the Shapiro-Wilk test $(P>0.05)$. The reliability of $V_{\text {last }}$ was assessed through the standard error of measurement (SEM), the CV and the ICC (model 3.1) with the corresponding 95\% confidence interval. Acceptable reliability was determined as a CV $<10 \%$ and a ICC $>0.70$ (Cormack et al., 2008). The association between the variables was quantified by the Pearson's correlation coefficient $(r)$ and the following scale was used to quantify the magnitude of the $r$ coefficient: trivial (.00-.09), small (.10-.29), moderate (.30-.49), large (.50-.69), very large (.70-.89), nearly perfect (.90-.99) and perfect (1.00) (Hopkins, Marshall, Batterham, \& Hanin, 2009). Paired sample's t tests, the Cohen's $d$ effect size (ES), and Bland-Altman plots were used to compare the magnitude of the variables. The following scale was used to interpret the magnitude of the ES: trivial $(<0.20)$, small $(0.20-0.59)$, moderate (0.60-1.19), large (1.20-1.99) and very large ( $\geq 2.00)$ (Hopkins et al., 2009). The reliability assessment was performed by means of a custom spreadsheet (Hopkins, 2000), while all other statistical analyses were performed using the software SPSS version 22.0 (SPSS, Chicago, IL, USA). Statistical significance was set at an alpha level of 0.05 .

\section{Results}

The reliability of $\mathrm{V}_{\text {last }}$ was unacceptable for both the paused $\mathrm{BP}(\mathrm{CV}=18.3 \%, \mathrm{ICC}=$ $0.58)$ and the touch-and-go BP $(\mathrm{CV}=20.4 \%, \mathrm{ICC}=0.48)($ Table 1$)$. The $\mathrm{V}_{1 \mathrm{RM}}$ was significantly higher than $\mathrm{V}_{\text {last }}$ during the paused BP $(P=0.028, \mathrm{ES}=0.30)$ and the touch-and-go BP $(P=0.020$, ES $=0.29)$, while the magnitude of the correlations between $\mathrm{V}_{1 \mathrm{RM}}$ and $\mathrm{V}_{\text {last }}$ was small for the paused BP $(r=0.18, P=0.083)$ and moderate for the touch-and-go BP $(r=0.37, P<0.001)$ (Figure 1). No significant differences between the BP variants were observed for $\mathrm{V}_{1 \mathrm{RM}}$ or $\mathrm{V}_{\text {last }}(P>0.80)$, while the correlations between the BP variants was trivial for $\mathrm{V}_{1 \mathrm{RM}}(r=-0.01, P=0.937)$ and moderate for $\mathrm{V}_{\text {last }}(r=0.46, P<0.001)$ (Figure 2). Bland-Altman plots revealed comparable systematic bias $\left(0.015-0.016 \mathrm{~m} \cdot \mathrm{s}^{-1}\right)$ and random errors $\left(0.054-0.057 \mathrm{~m} \cdot \mathrm{s}^{-1}\right)$ between $\mathrm{V}_{1 \mathrm{RM}}$ and $\mathrm{V}_{\text {last }}$ for both BP variants (Figure 3). A small and inverse relationship was observed between $V_{\text {last }}$ and the number of repetitions performed to failure $(r=-$ $0.23, P<0.001)$ and the 1 RM load $(r=-0.17, P=0.001)$, while moderate correlations were observed between $\mathrm{V}_{1 \mathrm{RM}}$ and the $1 \mathrm{RM}$ load $(r=-0.33, P<0.001)$ (Figure 4). Participants performed the sets of repetitions to failure against an average load of 82.1 $\pm 3.7 \%$ of $1 \mathrm{RM}$ and completed $6.9 \pm 2.3$ repetitions.

\section{[Table 1]}

Peer] reviewing PDF | (2019:11:43603:1:1:NEW 22 Jan 2020) 


\section{3 [Figure 1]}

244 [Figure 2]

245 [Figure 3]

246 [Figure 4]

247

248

\section{Discussion}

249

250

251

This study is the first to investigate the reliability of $V_{\text {last }}$ during the paused and touchand-go BP exercises, and explore the association between $\mathrm{V}_{1 \mathrm{RM}}$ and $\mathrm{V}_{\text {last }}$ within and between each BP variant. Additionally, this study determined the effect of the number of repetitions performed to failure on $V_{\text {last }}$, and the effect of $1 R M$ strength on $V_{1 R M}$ and $V_{\text {last }}$.

252

253

254

255 The main findings of the study revealed (I) low reliability for $V_{\text {last, }}$ (II) $V_{\text {last }}$ was significantly lower than $V_{1 R M}$, (III) no significant differences between the BP variants for $V_{1 R M}$ or $V_{\text {last }}$ (IV) larger associations between the BP variants for $V_{\text {last }}$ compared to $V_{1 R M}$, and $(V)$ a negative, albeit weak, association of $V_{\text {last }}$ with the number of repetitions performed to failure and the $1 R M$ strength. These results suggest that $V_{\text {last }}$ could be $a$ better indicator of the minimal velocity threshold during the BP exercise than $V_{1 R M}$ because $V_{\text {last }}$ was lower than $V_{1 R M}$ and it demonstrated a stronger relationship between the $\mathrm{BP}$ variants. However, the low reliability of $\mathrm{V}_{\text {last }}$, moderate association between the $B P$ variants for $V_{\text {last }}$, and the lack of differences between the $B P$ variants for the $V_{1 R M}$ or $V_{\text {last }}$ suggest that a general $V_{1 R M}$ could be more appropriate.

263

264

Previous studies have found that the magnitude of $V_{\text {last }}$ is not affected by the load applied during sets of repetitions to failure performed with the BP and back squat exercises (Izquierdo et al., 2006). However, no previous study had examined the reliability of $V_{\text {last }}$ in any resistance training exercise. Our results confirmed that $V_{\text {last }}$ is an unreliable metric for the 2 variants of the BP exercise examined in the present study. The reliability of $V_{\text {last }}(C V=18.3 \%$ and $20.4 \%$ for the paused and touch-and-go BP, respectively) was somewhat comparable to the reliability of the MCV associated with the 1RM load reported by Pestaña-Melero et al. (2018) (CV =13.9\% and $15.7 \%$ for the paused and touch-and-go BP, respectively). Therefore, it seems that neither the $V_{1 R M}$ nor the $V_{\text {last }}$ should be used on an individual basis since the within-subject variability of these variables is not meaningfully lower than the variability existing between subjects (Pestaña-Melero et al., 2018).

The generally low correlations between $\mathrm{V}_{1 \mathrm{RM}}$ and $\mathrm{V}_{\text {last }}$ for both BP variants $(r \leq$ 0.37 ) indicates that the value of one variable cannot be inferred from the other. Similarly, as it was previously shown by García-Ramos et al. (2018), no significant correlations were observed between the $2 \mathrm{BP}$ variants for $\mathrm{V}_{1 \mathrm{RM}}(r=-0.01)$. Only the value of $\mathrm{V}_{\text {last }}$ was moderately correlated between the $2 \mathrm{BP}$ variants $(r=0.46)$, revealing that participants with a higher $V_{\text {last }}$ in one BP variant also tended to have a higher $V_{\text {last }}$ in the other BP variant. These results provide additional support for the use of the same minimal velocity threshold (i.e. $V_{1 R M}$ ) for all participants when predicting the 1RM from the individualized L-V relationship during the BP exercise performed in a Smith machine. The lack of differences between the BP variants for the magnitudes of $V_{1 R M}$ and $V_{\text {last }}$ simplifies this approach because the same minimal velocity threshold could be used for both BP variants. According to the results of this and previous studies, the 
289 minimal velocity threshold should be set at $0.17 \mathrm{~m} \cdot \mathrm{s}^{-1}$ (García-Ramos et al., 2018; 290 García-Ramos et al., 2019; González-Badillo \& Sánchez-Medina, 2010; Sánchez291 Medina, González-Badillo, Pérez, \& Pallarés, 2014). However, Helms et al. (2017) 292 reported in 15 powerlifters (12 men and 3 women) a lower $V_{1 R M}$ during the free-weight BP exercise that was performed using a "press" command to simulate a powerlifting competition. Therefore, additional research should be conducted with different variants of the free-weight BP and more trained populations to elucidate whether the minimal velocity threshold recommended in the present study can also be applicable to these conditions.

In the present study we also explored the influence of $1 \mathrm{RM}$ strength on the values of $V_{1 R M}$ and $V_{\text {last. }}$. Based on the lower $V_{1 R M}$ reported by Helms et al. (2017) in

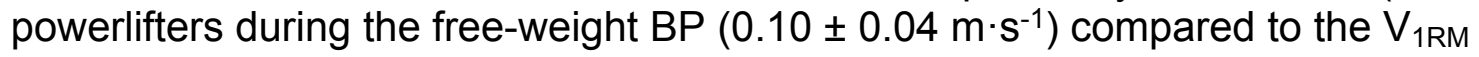
reported by Loturco et al. (2017) in rugby players and combat athletes $\left(0.17 \mathrm{~m} \cdot \mathrm{s}^{-1}\right)$, we hypothesized that the $1 \mathrm{RM}$ strength would be negatively associated with $\mathrm{V}_{1 \mathrm{RM}}$ and $\mathrm{V}_{\text {last. }}$ This hypothesis was somewhat supported with a significant, albeit weak, negative correlation between the $1 R M$ load and $V_{1 R M}(r=-0.33)$ and $V_{\text {last }}(r=-0.17)$. This result is also in line with the findings of Ormsbee et al. (2019) who reported a slower $V_{1 R M}$ for experienced benchers $\left(0.14 \pm 0.04 \mathrm{~m} \cdot \mathrm{s}^{-1}\right)$ compared to novice benchers $(0.20 \pm 0.05$ $\left.\mathrm{m} \cdot \mathrm{s}^{-1}\right)$. Therefore, although based on these results a slightly lower minimal velocity threshold could be obtained by stronger subjects, the low reliability and low correlations found in the present study for $V_{1 R M}$ and $V_{\text {last }}$ suggest that it may not be necessary to modify the minimal velocity threshold based on the $1 \mathrm{RM}$ strength values when the bench press is performed in a Smith machine. Finally, we also explored the influence of the number of repetitions performed during the sets to failure on $V_{\text {last }}$, and we observed a relatively weak negative association $(r=-0.23)$, indicating that a slightly lower $V_{\text {last }}$ can be obtained when higher number of repetitions are performed. Therefore, in the case that coaches are interested in determining $V_{\text {last }}$, we recommended that $V_{\text {last }}$ is derived from a set to failure of at least 5 repetitions.

A limitation of the present study is that the $1 \mathrm{RM}$ was directly assessed only once for each BP variant and, therefore, it was not possible to compare the reliability between $V_{1 R M}$ and $V_{\text {last }}$. However, the lower correlations between the BP variants observed in this study for $V_{1 R M}$ compared to $V_{\text {last }}$ suggest that the $V_{1 R M}$ could also be less reliable than $V_{\text {last. }}$ This would corroborate the results of previous studies that have reported a poor reliability for the $\mathrm{V}_{1 \mathrm{RM}}$ during the BP (Pestaña-Melero et al., 2018) and other resistance training exercises such as the back squat (Banyard, Nosaka \& Haff, 2017), deadlift (Ruf, Chery \& Taylor, 2018), or bench pull (García-Ramos et al., 2019). Another limitation is that the BP was performed in a Smith machine, while the vast majority of athletes use the free-weight BP during their resistance training programs. In the current study, the Smith machine was used to simplify the testing procedure and to improve the reproducibility of velocity readings. Thus, it is plausible that the reliability of $V_{\text {last }}$ and $V_{1 R M}$ may be even lower if the BP is performed with free-weights due to the additional control required and horizontal movements characterizing the free weight BP. Finally, the sample of this study was composed of males with a mean of 1.3 years of experience with the BP exercise and, therefore, it is possible that the accuracy of $V_{1 \mathrm{RM}}$ and $\mathrm{V}_{\text {last }}$ 
335

336

337

338

339

340

341

342

343

344

345

346

347

348

349

350

351

352

353

354

355

356

357

358

359

360

361

362

363

364

365

366

367

368

369

370

371

372

373

374

375

376

377

378

379

380

could increase when testing participants with more experience. Future studies should compare the magnitude of $\mathrm{V}_{1 \mathrm{RM}}$ and $\mathrm{V}_{\text {last }}$ as well as the reliability of these variables between individuals with different training backgrounds to shed light on this topic.

\section{Conclusions}

The reliability of $\mathrm{V}_{\text {last }}$ was below the threshold of acceptable reliability for both BP variants. $V_{\text {last }}$ was always significantly lower than $V_{1 R M}$, while no significant differences between the BP variants were observed for $\mathrm{V}_{1 \mathrm{RM}}$ or $\mathrm{V}_{\text {last }}$. $\mathrm{V}_{\text {last }}$ was more correlated between the $\mathrm{BP}$ variants than $\mathrm{V}_{1 \mathrm{RM}}$. The correlations between $\mathrm{V}_{1 \mathrm{RM}}$ and $\mathrm{V}_{\text {last }}$ ranged from small (paused BP) to moderate (touch-and-go BP). An inverse, but generally weak, association was observed between $V_{\text {last }}$ and the number of repetitions performed to failure and the 1RM load, as well as between the $V_{1 \mathrm{RM}}$ and the 1RM load. Therefore, even though $V_{\text {last }}$ could be a more appropriate indicator of the minimal velocity threshold than $\mathrm{V}_{1 \mathrm{RM}}$, our results (i.e. low reliability, lack of differences in magnitude and only moderate association between the BP variants) also suggest that a general $V_{1 \mathrm{RM}}$ could be more appropriate to estimate the $1 \mathrm{RM}$ during the BP exercise performed in a Smith machine.

\section{Acknowledgements}

We would like to thank all the participants who volunteered to participate in this study.

\section{References}

Banyard HG, Nosaka K, Haff GG. 2017. Reliability and validity of the load-velocity relationship to predict the 1RM back squat. Journal of Strength and Conditioning Research 31:1897-1904. DOI: 10.1519/JSC.0000000000001657.

Cormack SJ, Newton RU, McGuigan MR, Doyle TLA. 2008. Reliability of measures obtained during single and repeated countermovement jumps. International Journal of Sports Physiology and Performance 3:131-144. DOI: 10.1123/ijspp.3.2.131.

Garcia-Ramos A, Barboza-Gonzalez P, Ulloa-Diaz D, Rodriguez-Perea A, MartinezGarcia D, Guede-Rojas F, Hinojosa-Riveros H, Chirosa-Rios LJ, Cuevas-Aburto J, Janicijevic D, Weakley J. 2019. Reliability and validity of different methods of estimating the one-repetition maximum during the free-weight prone bench pull exercise. Journal of Sports Sciences 37:2205-2212. DOI:

10.1080/02640414.2019.1626071.

García-Ramos A, Haff GG, Pestaña-Melero FL, Pérez-Castilla A, Rojas FJ, BalsalobreFernández C, Jaric S. 2018a. Feasibility of the 2-point method for determining the 1-repetition maximum in the bench press exercise. International Journal of Sports Physiology and Performance 13:474-481. DOI: 10.1123/ijspp.2017-0374.

García-Ramos A, Jaric S. 2018. Two-point method: a quick and fatigue-free procedure for assessment of muscle mechanical capacities and the one-repetition maximum. Strength and Conditioning Journal 40:54-66. DOI: 10.1519/SSC.0000000000000359.

García-Ramos A, Pestaña-Melero FL, Pérez-Castilla A, Rojas FJ, Haff GG. $2018 b$. Differences in the load-velocity profile between 4 bench press variants. International Journal of Sports Physiology and Performance 13:326-331. DOI: 10.1123/ijspp.2017-0158.

Peer) reviewing PDF | (2019:11:43603:1:1:NEW 22 Jan 2020) 
381

382

383

384

385

386

387

388

389

390

391

392

393

394

395

396

397

398

399

400

401

402

403

404

405

406

407

408

409

410

411

412

413

414

415

416

417

418

419

420

421

422

423

424

425

426

García-Ramos A, Pestaña-Melero FL, Pérez-Castilla A, Rojas FJ, Haff GG. 2018c. Mean velocity vs. mean propulsive velocity vs. peak velocity: which variable determines bench press relative load with higher reliability? Journal of Strength and Conditioning Research 32:1273-1279. DOI: 10.1519/jsc.0000000000001998.

García-Ramos A, Ulloa-Díaz D, Barboza-González P, Rodríguez-Perea Á, MartínezGarcía D, Quidel-Catrilelbún M, Guede-Rojas F, Cuevas-Aburto J, Janicijevic D, Weakley J. 2019. Assessment of the load-velocity profile in the free-weight prone bench pull exercise through different velocity variables and regression models. PloS one 14:e0212085-e0212085. DOI: 10.1371/journal.pone.0212085.

González-Badillo J, Sánchez-Medina L. 2010. Movement velocity as a measure of loading intensity in resistance training. International Journal of Sports Medicine 31:347-352. DOI: 10.1055/s-0030-1248333.

Helms ER, Storey A, Cross MR, Brown SR, Lenetsky S, Ramsay H, Dillen C, Zourdos MC. 2017. RPE and velocity relationships for the back squat, bench press, and deadlift in powerlifters. Journal of Strength and Conditioning Research 31:292-297. DOI: $10.1519 / J S C .0000000000001517$.

Hopkins W. 2000.Calculations for reliability (Excel spreedsheet). Available at http://www.sportsci.org/resource/stats/relycalc.html\%7B\#\%7Dexcel

Hopkins WG, Marshall SW, Batterham AM, Hanin J. 2009. Progressive statistics for studies in sports medicine and exercise science. Medicine and Science in Sports and Exercise 41:3-13. DOI: 10.1249/MSS.0b013e31818cb278.

Izquierdo M, González-Badillo JJ, Häkkinen K, Ibáñez J, Kraemer WJ, Altadill A, Eslava J, Gorostiaga EM. 2006. Effect of loading on unintentional lifting velocity declines during single sets of repetitions to failure during upper and lower extremity muscle actions. International Journal of Sports Medicine 27:718-724. DOI: 10.1055/s2005-872825.

Lake J, Naworynsky D, Duncan F, Jackson and M. 2017. Comparison of different minimal velocity thresholds to establish deadlift one repetition maximum. Sports 5:70. DOI: https://doi.org/10.3390/sports5030070.

Loturco I, Kobal R, Moraes JE, Kitamura K, Cal Abad CC, Pereira LA, Nakamura FY. 2017. Predicting the maximum dynamic strength in bench press: The high precision of the bar velocity approach. Journal of strength and conditioning research 31:1127-1131. DOI: 10.1519/JSC.0000000000001670.

McBurnie AJ, Allen KP, Garry M, Martin M, Thomas D, Jones PA, Comfort P, McMahon JJ. 2019. The benefits and limitations of predicting one repetition maximum using the load-velocity relationship. Strength \& Conditioning Journal. In press.

Ormsbee MJ, Carzoli JP, Klemp A, Allman BR, Zourdos MC, Kim J-S, Panton LB. 2019. Efficacy of the repetitions in reserve-based rating of perceived exertion for the bench press in experienced and novice benchers. Journal of Strength and Conditioning Research 33:337-345. DOI: 10.1519/JSC.0000000000001901.

Pallarés JG, Sánchez-Medina L, Pérez CE, De La Cruz-Sánchez E, Mora-Rodriguez R. 2014. Imposing a pause between the eccentric and concentric phases increases the reliability of isoinertial strength assessments. Journal of sports sciences 32:1165-75. DOI: 10.1080/02640414.2014.889844.

Pérez-Castilla A, Comfort P, McMahon J, Pestaña-Melero F, García-Ramos A. 2018. Comparison of the force-, velocity- and power-time curves between the concentric- 
427 only and eccentric-concentric bench press exercises. The Journal of Strength and $428 \quad$ Conditioning Research. In press.

Pestaña-Melero F, Haff GG, Rojas FJ, Pérez-Castilla A, García-Ramos A. 2018. Reliability of the load-velocity relationship obtained through linear and polynomial regression models to predict the one-repetition maximum load. Journal of Applied Biomechanics 34:184-190. DOI: 10.1123/jab.2017-0266.

Ruf L, Chery C, Taylor K-L. 2018. Validity and reliability of the load-velocity relationship to predict the one-repetition maximum in deadlift. Journal of strength and conditioning research 32:681-689. DOI: 10.1519/JSC.0000000000002369.

Sánchez-Medina L, González-Badillo JJ, Pérez CE, Pallarés JG. 2014. Velocity- and power-load relationships of the bench pull vs. bench press exercises. International 439 Journal of Sports Medicine 35:209-216. DOI: 10.1055/s-0033-1351252. 


\section{Figure legends}

441 Figure 1. Association ( $A$ and $C$ ) and comparison ( $B$ and $D$ ) between the velocity of the 442 1-repetition maximum $\left(\mathrm{V}_{1 \mathrm{RM}}\right)$ and the velocity achieved during the last repetition of sets 443 to failure $\left(V_{\text {last }}\right)$ during the paused $(A$ and $B)$ and touch-and-go $(C$ and $D)$ bench press 444 (BP) exercises. $r$, Pearson's correlation coefficient; $p$, $p$-value obtained from paired 445 sample's $t$ tests; $E S$, Cohen's $d$ effect size $\left[\left(V_{1 R M}\right.\right.$ mean $-V_{\text {last }}$ mean $\left.) / S D b o t h\right]$.

Figure 2. Association ( $A$ and $C$ ) and comparison ( $B$ and $D$ ) between the paused bench 448 press (BP) and the touch-and-go BP for the velocity of the 1-repetition maximum ( $\mathrm{V}_{1 \mathrm{RM}}$; $A$ and $B)$ and the velocity achieved during the last repetition of sets to failure ( $V_{\text {last }} ; C$ and D). $r$, Pearson's correlation coefficient; $p, p$-value obtained from paired sample's $t$ tests; ES, Cohen's $d$ effect size [(paused BP mean - touch-and-go BP mean)/SDboth].

Figure 3. Bland-Altman plots showing the differences between the velocity of the 1repetition maximum $\left(\mathrm{V}_{1 \mathrm{RM}}\right)$ and the velocity achieved during the last repetition of sets to failure $\left(V_{\text {last }}\right)$ during the paused bench press (BP) (A) and the touch-and-go BP (B). Each plot depicts the systematic bias and $95 \%$ limits of agreement $( \pm 1.96 \mathrm{SD}$; dashed lines), along with the regression line (solid line). The systematic bias \pm random error together with the strength of the relationship $\left(r^{2}\right)$ are depicted in each plot.

Figure 4. Relationship between the number of repetitions performed to failure and $V_{\text {last }}$ (A), 1-repetition maximum and $V_{\text {last }}(B)$, and 1-repetition maximum and $V_{1 R M}(C) . V_{\text {last, }}$, velocity of the last repetition performed during sets of repetitions to failure; $V_{1 R M}$, velocity 464 achieved during the 1-repetition maximum trial. The regression equation and the Pearson's correlation coefficient $(r)$ are depicted. 


\section{Figure 1}

Association and comparison between the velocity of the 1-repetition maximum and the velocity achieved during the last repetition of sets to failure

Association (left hand panels) and comparison (right hand panels) between the velocity of the 1-repetition maximum $\left(\mathrm{V}_{1 \mathrm{RM}}\right)$ and the velocity achieved during the last repetition of sets to failure $\left(\mathrm{V}_{\text {last }}\right)$ during the paused (upper panels) and touch-and-go (lower panels) bench press (BP) exercises. $r$, Pearson's correlation coefficient; $p, p$-value obtained from paired sample's $t$ tests; ES, Cohen's $d$ effect size $\left[\left(\mathrm{V}_{1 \mathrm{RM}}\right.\right.$ mean - $\mathrm{V}_{\text {last }}$ mean $) / \mathrm{SDb}$ oth $]$.
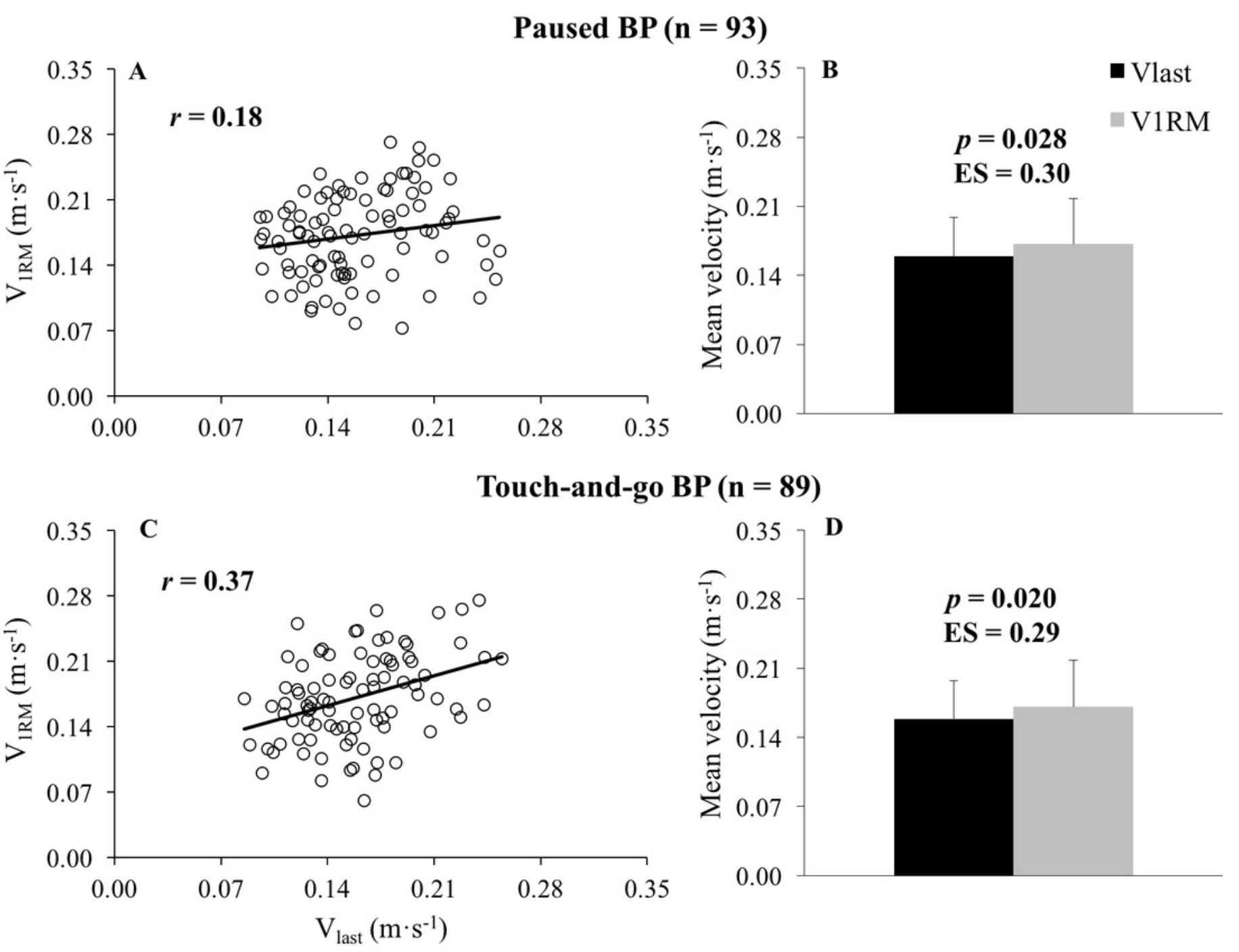


\section{Figure 2}

Association and comparison between the paused bench press and the touch-and-go bench press for the velocity of the 1-repetition maximum and the velocity achieved during the last repetition of sets to failure

Association (left hand panels) and comparison (right hand panels) between the paused bench press (BP) and the touch-and-go BP for the velocity of the 1-repetition maximum $\left(\mathrm{V}_{\text {1Rм}}\right.$; upper panels) and the velocity achieved during the last repetition of sets to failure $\left(\mathrm{V}_{\text {last }} ;\right.$ lower panels). $r$, Pearson's correlation coefficient; $p, p$-value obtained from paired sample's t tests; ES, Cohen's $d$ effect size [(paused BP mean - touch-and-go BP mean)/SDboth]. 

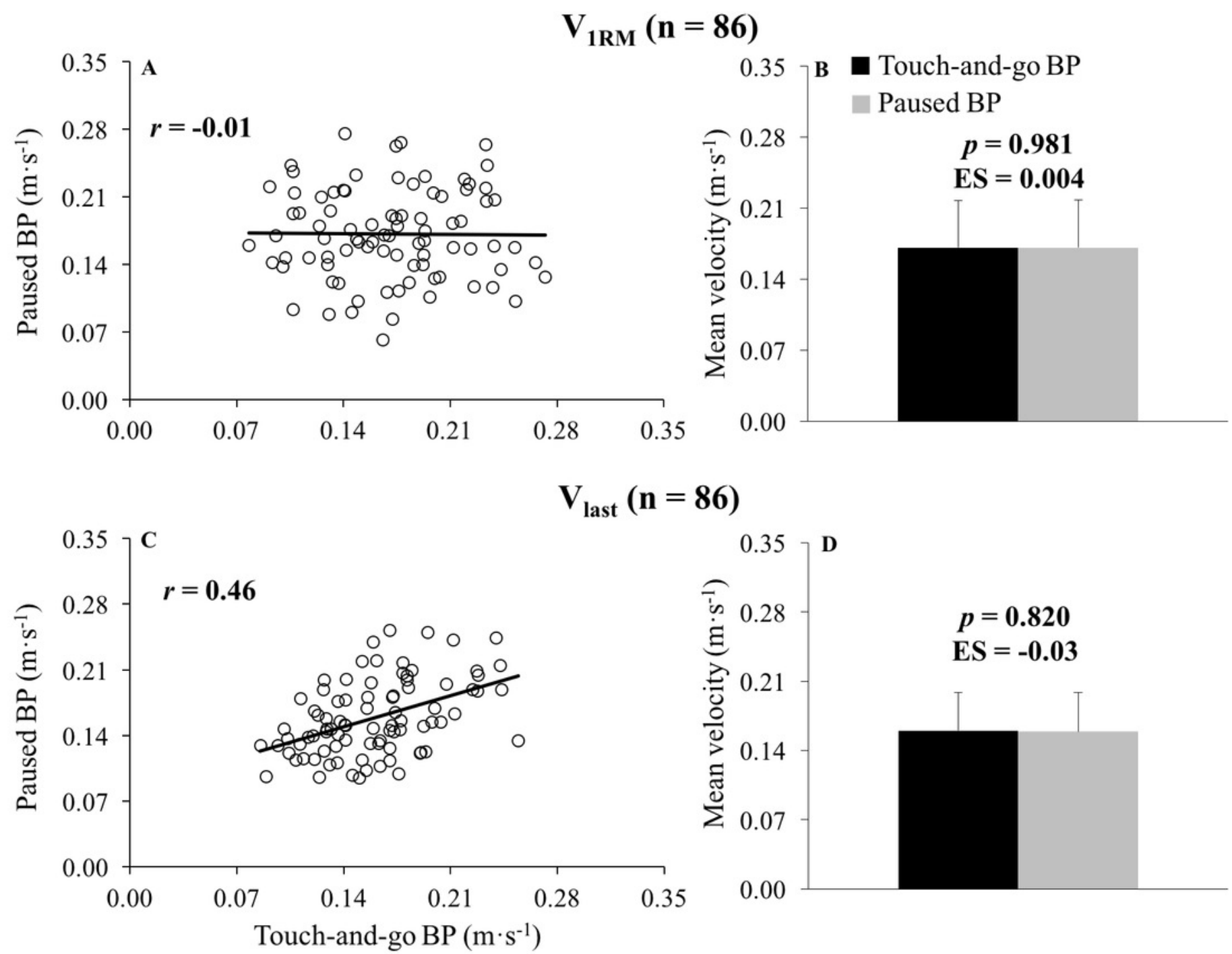


\section{Figure 3}

Differences between the velocity of the 1-repetition maximum and the velocity achieved during the last repetition of sets to failure during the paused bench press (BP) and the touch-and-go BP.

Bland-Altman plots showing the differences between the velocity of the 1-repetition maximum $\left(\mathrm{V}_{1 \mathrm{RM}}\right)$ and the velocity achieved during the last repetition of sets to failure $\left(\mathrm{V}_{\text {last }}\right)$ during the paused bench press (BP) (upper panel) and the touch-and-go BP (lower panel). Each plot depicts the systematic bias and $95 \%$ limits of agreement ( $\pm 1.96 \mathrm{SD}$; dashed lines), along with the regression line (solid line). The systematic bias \pm random error together with the strength of the relationship $\left(r^{2}\right)$ are depicted in each plot. 

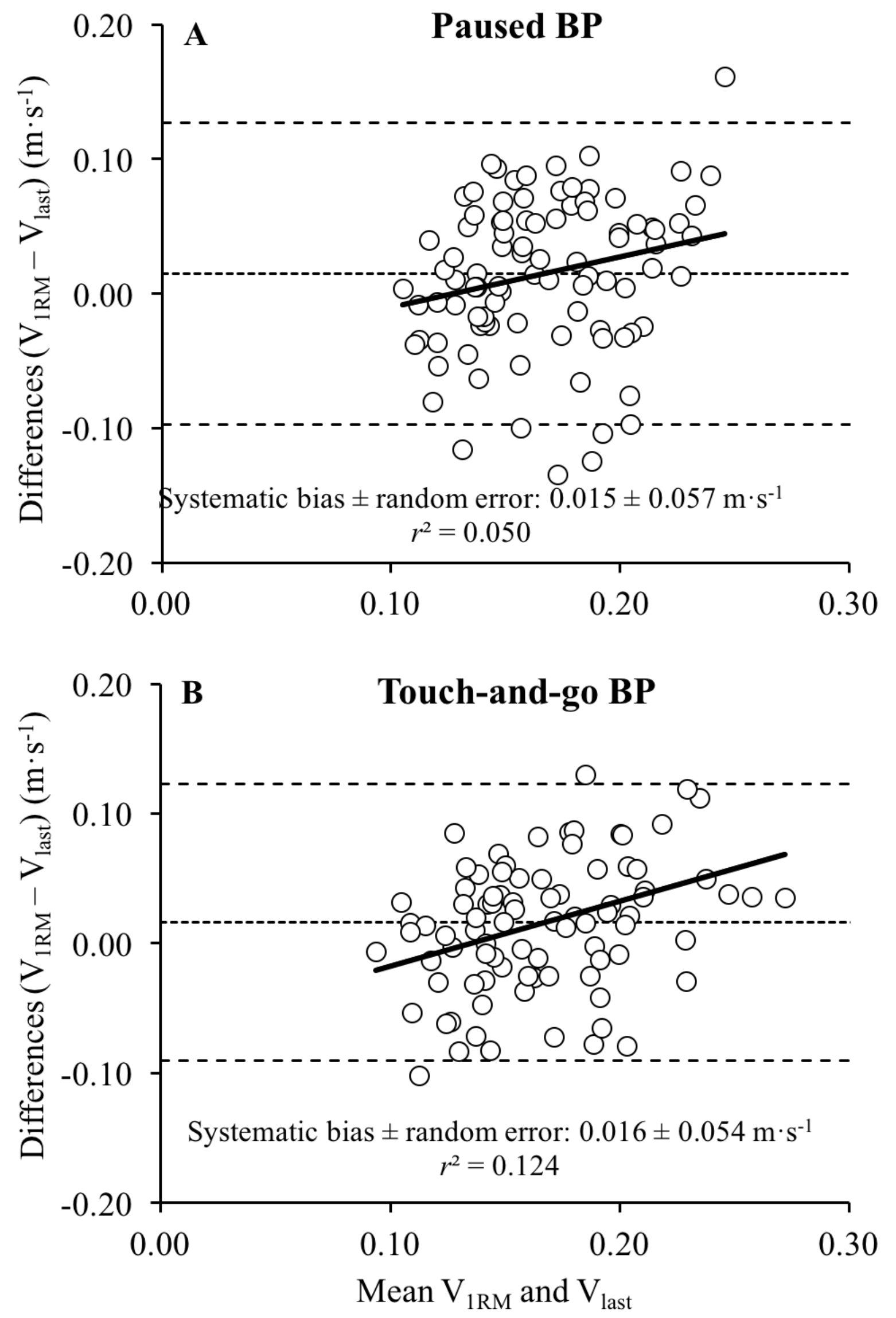


\section{Figure 4}

Relationship between the number of repetitions performed to failure and velocity of the last repetition performed during sets of repetitions to failure $\left(V_{\text {lass }}\right)$, 1-repetition maximum and $V_{\text {last }}$ and 1-repetition maximum and velocity

Relationship between the number of repetitions performed to failure and $V_{\text {last }}$ (upper panel), 1-repetition maximum and $\mathrm{V}_{\text {last }}$ (middle panel), and 1-repetition maximum and $\mathrm{V}_{1 \mathrm{RM}}$ (lower panel). $V_{\text {last }}$ velocity of the last repetition performed during sets of repetitions to failure; $V_{1 R M}$, velocity achieved during the 1-repetition maximum trial. The regression equation and the Pearson's correlation coefficient $(r)$ are depicted 

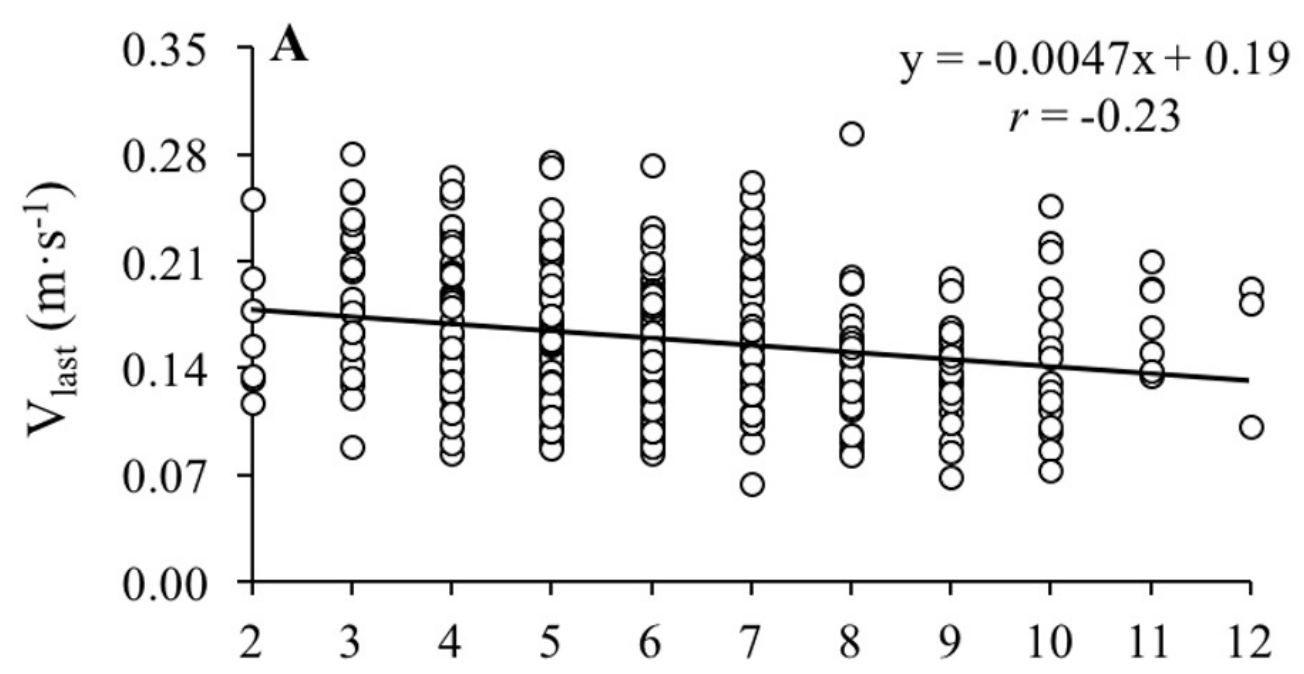

\section{Number of repetitions performed}
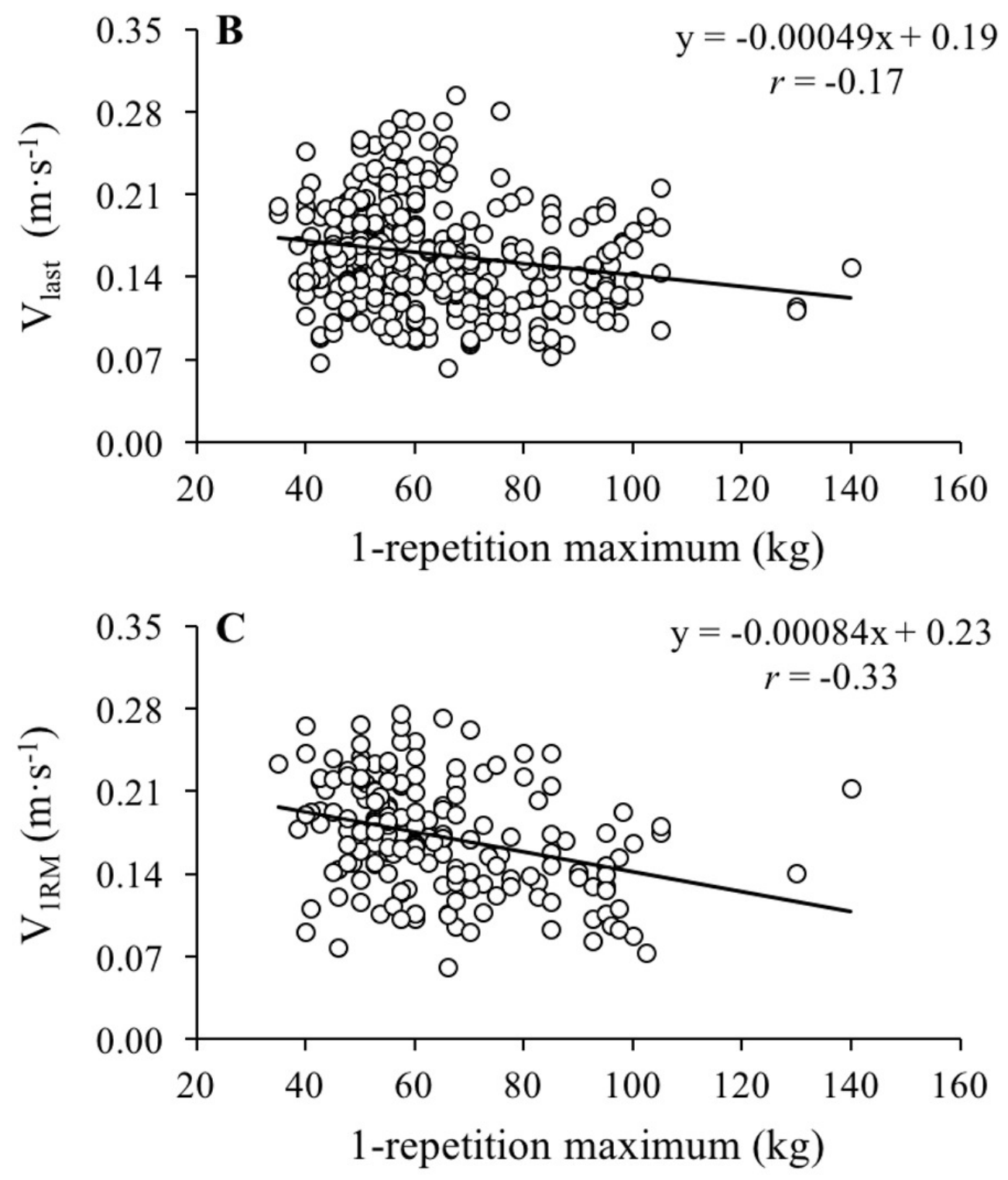


\section{Table $\mathbf{1}$ (on next page)}

Reliability of mean concentric velocity values achieved during the last repetition of sets to failure during the paused and touch-and-go bench press exercises

$\mathrm{BP}$, bench press; $p$, $\mathrm{p}$-value; $\mathrm{CV}$, coefficient of variation; ICC, intraclass correlation coefficient; SEM, standard error of measurement; $95 \% \mathrm{Cl}, 95 \%$ confidence interval 
1 Table 1. Reliability of mean concentric velocity values achieved during the last repetition of sets 2 to failure during the paused and touch-and-go bench press exercises.

3

\begin{tabular}{|c|c|c|c|c|c|c|}
\hline Exercise & $\begin{array}{l}\text { Set } 1 \\
\left(\mathrm{~m} \cdot \mathrm{s}^{-1}\right)\end{array}$ & $\begin{array}{l}\text { Set } 2 \\
\left(\mathrm{~m} \cdot \mathrm{s}^{-1}\right)\end{array}$ & $p$ & $\begin{array}{c}\mathrm{CV} \\
(95 \% \mathrm{CI}) \\
\end{array}$ & $\begin{array}{c}\text { ICC } \\
(95 \% \mathrm{CI})\end{array}$ & $\begin{array}{l}\text { SEM } \\
\left(\mathrm{m} \cdot \mathrm{s}^{-1}\right)\end{array}$ \\
\hline $\begin{array}{c}\text { Paused BP } \\
(n=93)\end{array}$ & $\begin{array}{c}0.161 \\
(0.045)\end{array}$ & $\begin{array}{c}0.157 \\
(0.045) \\
\end{array}$ & 0.303 & $\begin{array}{c}18.3 \% \\
(16.0 \%, 21.4 \%)\end{array}$ & $\begin{array}{c}0.58 \\
(0.43,0.70)\end{array}$ & 0.029 \\
\hline $\begin{array}{l}\text { Touch-and-go BP } \\
(\mathrm{n}=89)\end{array}$ & $\begin{array}{c}0.162 \\
(0.046)\end{array}$ & $\begin{array}{c}0.156 \\
(0.043) \\
\end{array}$ & 0.266 & $\begin{array}{c}20.4 \% \\
(17.8 \%, 24.0 \%)\end{array}$ & $\begin{array}{c}0.48 \\
(0.30,0.62)\end{array}$ & 0.032 \\
\hline
\end{tabular}

4 BP, bench press; $p$, $\mathrm{p}$-value; $\mathrm{CV}$, coefficient of variation; ICC, intraclass correlation coefficient; 5 SEM, standard error of measurement; $95 \%$ CI, 95\% confidence interval. 